\title{
Motivos y reacciones ante la infidelidad en población universitaria
}

\author{
MARÍA ISABEL LÓPEZ BELTRÁN \\ al189575@uji.es \\ MARÍA Amparo PÉREZ BARberÁ \\ al189629@uji.es \\ MARÍA BELÉn PIQUER BARRACHINA \\ al209517@uji.es \\ RAFAEL BALLESTER-ARNAL \\ rballest@uji.es
}

\section{Resumen}

Introducción: El objetivo del presente estudio es analizar cuáles son los motivos actuales más prevalentes por los que la población universitaria comete una infidelidad, así como examinar las posibles reacciones y diferencias en función del género. Método: La muestra está compuesta por 194 estudiantes con una edad promedio de 20,8 años ( $D T=2,06$ ), equiparados por sexo. Los participantes cumplimentaron un instrumento de elaboración propia de forma voluntaria y confidencial. Resultados: El 27,5\% declara haber sido infiel a alguna de sus parejas, de los cuales el 38,5\% ha admitido que cometió la infidelidad porque el vínculo emocional era poco intenso. En cuanto a las diferencias de género, solo hay diferencias significativas a favor de las mujeres, las cuales han respondido en mayor medida que los hombres que fueron infieles porque estaban bajo los efectos del alcohol o drogas $\left(x^{2}=6,422 ; p \leq, 011\right)$. La reacción más común ante una infidelidad $(63,6 \%)$ sería cortar la relación. Por otro lado, el 34,6 \% afirma que su pareja le ha sido infiel. En relación a esto, existe una relación positiva $(r=0,259 ; p \leq, 000)$ entre ser infiel y que la pareja le haya sido infiel. Discusión: En consonancia con estudios similares, el principal motivo por el que se comete una infidelidad parece ser la fragilidad y las carencias afectivas del vínculo emocional de la pareja, siendo escasas las diferencias de género en cuanto a motivos para ser infiel.

Palabras clave: infidelidad, motivos, género, pareja, universitarios.

\section{Abstract}

Introduction: The objective of this study is to analyse what are today's most prevalent reasons why the university population commits infidelity and to discuss possible reactions to it and also gender differences. Method: The sample consisted of 194 students, whose average age was 20.79 years $(S D=2.06)$ and were sex-matched. Participants completed a voluntary and confidential instrument that the authors developed. Re- 
sults: Of the whole sample, $27.50 \%$ reported having never been unfaithful to their partners, and $38.50 \%$ admitted that they had committed infidelity because the emotional bond was not intense enough. The gender differences found were significant only for females, who responded to a greater extent than males that they had been unfaithful because they were under the influence of alcohol or drugs $\left(X^{2}=6.422 ; p=.011\right)$. The commonest reaction to infidelity $(63.60 \%)$ was to break the relationship. Moreover, $34.60 \%$ of the sample stated that their partner had been unfaithful, in relation to which a positive relationship $(r=0.259 ; p=.000)$ was observed between being unfaithful and if one's partner had been unfaithful. Discussion: In line with similar studies, the main reason for committing infidelity seemed to be fragility and the emotional deprivation of an emotional bond between the couple. Virtually no gender differences in the reasons for being unfaithful were found.

Keywords: infidelity, reasons, gender, couple, university

\section{Introducción}

La exposición a la infidelidad es algo común en nuestra sociedad, ya sea experimentada en primera persona o a través de amistades o incluso es un tema frecuente en los medios de comunicación, donde se puede apreciar una amplia variedad de formas de cometer una infidelidad (Brogdon, Fitzwater y Johnson, 2006). En la cultura occidental hay una fuerte exigencia de exclusividad en las relaciones de pareja, especialmente en el terreno sexual, y cualquier pérdida de esa exclusividad es considerada como una deslealtad (Vanegas, 2011). En las últimas décadas, se han realizado algunas conceptualizaciones acerca de la infidelidad, las cuales incluyen diversos aspectos, como la forma en la que se lleva a cabo la infidelidad, los motivos o las consecuencias que de esta se derivan.

La infidelidad podría considerarse como un abuso de la confianza dentro de una relación de pareja a través de un acto sexual o emocional con otra persona, rompiendo la exclusividad romántica/emocional o sexual e incumpliendo las normas y expectativas que se establecen dentro de la pareja (Blow y Hartnet, 2005). Algunos autores han postulado teorías evolutivas que podrían encontrarse en la base de la naturaleza de la infidelidad. En particular, la teoría de la inversión parental (Trivers, 1972) afirma que los hombres y las mujeres presentan estrategias reproductivas diferentes con el fin de obtener unos beneficios más adaptativos para cada sexo, buscando los hombres relaciones más oportunistas y siendo las mujeres más selectivas en sus relaciones: es decir, puesto que el objetivo del hombre sería tener una mayor progenie, él trata de tener el mayor número posible de encuentros sexuales, mientras que la mujer es más selectiva en sus encuentros sexuales con el fin de obtener una mayor protección y estabilidad para sus descendientes. Por otro lado, diversas investigaciones han estudiado las razones de tipo ontogenético que llevan a cometer una infidelidad. Algunas de las razones encontradas son, por ejemplo, la infelicidad en la pareja, el aburrimiento, la excitación, la falta de atracción, los estados de ánimo negativos, la venganza, la lujuria, la falta de comunicación en la pareja o incluso las relaciones abiertas (Brand, Markey, Mills y Hodges, 2007; Mark, Janssen y Milhausen, 2011; McAnulty y Brineman, 2007; Weiser, Lalasz, Weigel y Evans, 2014). Así mismo, a pesar de que las razones que llevan a cometer una infidelidad son similares e incluso, en muchas ocasiones, compartidas por hombres y mujeres, también se han informado algunas diferencias, como que los hombres son más propensos a dejarse llevar por 
el impulso mientras que las mujeres lo suelen hacer más debido a la insatisfacción con su pareja (Brand y cols., 2007; Haden y Hojjat, 2006; Shackelford y Goetz, 2008; Tidwell y Eastwick, 2013).

Siguiendo por esta misma línea, también se ha hablado de las consecuencias y del impacto que tiene la infidelidad en las relaciones de pareja, como el divorcio en el caso de los matrimonios o la ruptura en el caso de las parejas, el cambio de pareja o el desamor, así como las posibles diferencias de género en la reacción ante una infidelidad. En referencia a las mujeres, algunas de las reacciones más destacadas son la ruptura de la pareja o el distanciamiento de la misma; los hombres, por su parte, son más propensos a responder ante una infidelidad con violencia (Brand y cols., 2007; Weiser y cols., 2014).

Dada la gran variedad en referencia a los motivos y reacciones ante la infidelidad encontrados en la literatura, la presente investigación tiene por objetivo principal estudiar los motivos que llevan a la población universitaria a cometer una infidelidad así como las reacciones más frecuentes ante la misma. De este modo, podrían esclarecerse algunos aspectos relacionados con la conducta y la naturaleza de la infidelidad con el objetivo de establecer una base empírica que pueda resultar de ayuda en la práctica clínica en parejas.

\section{Método}

\section{Participantes}

Se seleccionaron un total de 194 participantes con un rango de edad comprendido entre 18 y 26 años ( $M=20,8$; DT = 2,06). De esta muestra empleada, 97 participantes $(50 \%)$ eran mujeres y 97 (50\%) eran hombres. En cuanto a la nacionalidad de los participantes, se encontraron 9 nacionalidades distintas, siendo la más mentada la española $(85,1 \%)$, seguida de la colombiana $(3,1 \%)$. También se registraron datos sobre la orientación sexual, con 140 participantes $(72,2 \%)$ heterosexuales, $34(17,5 \%)$ bisexuales y $20(10,3 \%)$ homosexuales. Por otra parte, en cuanto a la religión, el $33,9 \%$ de los participantes eran creyentes.

\section{Procedimiento}

En lo que se refiere al procedimiento de recogida de la muestra, se realizaron dos recogidas diferentes. La primera, de forma presencial durante 4 días por las distintas facultades de la Universitat Jaume I, y la segunda opción fue mediante el cuestionario en formato online. En ambos casos, los participantes fueron informados de los objetivos de la investigación y de la confidencialidad con la que se tratarían los datos.

\section{Instrumento de evaluación}

Para llevar a cabo el presente estudio ha sido necesaria la creación de un cuestionario adaptado a los objetivos de la investigación. Para ello, se han tomado como referencia algunos cuestionarios (aunque no validados) que se han centrado en evaluar aspectos similares, como es el caso de The infidelity questionnaire (Nagurney y Thornton, 2011) o The sexual behavior questionnaire (Norona, 2013).

El instrumento final empleado para la recogida de la información consiste en un cuestionario de elaboración propia compuesto por dos partes diferenciadas. Al comienzo, contiene 
información de tipo demográfica (sexo, edad, nacionalidad, ocupación, orientación sexual, religión). Además, se incluye información sobre relaciones de pareja, pareja en la actualidad o el número de parejas que ha tenido y su duración. Para esta investigación, únicamente se tendrá en cuenta la información obtenida de la primera parte, la cual incluye 17 ítems relacionados con información sobre, por ejemplo, si alguna vez ha sido infiel, los motivos, el tipo de infidelidad, el uso del preservativo en esa situación, las consecuencias de la infidelidad, si alguna vez le han sido infiel, los celos, las conductas de control sobre su pareja o la utilización de los servicios de un/a trabajador/a sexual.

\section{Análisis de datos}

Se han realizado análisis descriptivos de frecuencias, tablas de contingencia, análisis de Chi-cuadrado y correlaciones. Los análisis estadísticos se realizaron mediante el programa estadístico SPS Statistics versión 23.

\section{Resultados}

\section{Prevalencia de la infidelidad realizada y recibida}

De los 194 participantes, un 72,5\% declara no haber sido infiel a sus parejas nunca, un $24,4 \%$ había sido algunas veces infiel, un 2,1 \% había sido bastantes veces y un 1,0 \% había sido infiel muchas veces. De las personas que habían sido infieles, el 30,2 \% expresó que fue con alguien de su mismo sexo y un $80,8 \%$ con una persona de distinto sexo. Además, un $34,6 \%$ de los participantes han sido víctimas de una infidelidad.

Por otra parte, en cuanto a la prevalencia de la infidelidad en función del sexo, el $31 \%$ de los hombres y el $24 \%$ de las mujeres han sido infieles a sus parejas, sin establecerse diferencias significativas entre los grupos. En relación con esto, el 30,9\% de los hombres y el $38,3 \%$ de las mujeres han sido víctimas de una infidelidad. En lo que se refiere a la prevalencia de la infidelidad en función de la orientación sexual, se ha encontrado que las personas con una orientación sexual homosexual son significativamente más infieles que las personas con orientación heterosexual y bisexual $\left(x^{2}=20,312 ; p \leq, 002\right)$.

\section{Prevalencia de los distintos motivos para cometer una infidelidad}

Teniendo en cuenta los resultados aportados por estudios anteriores similares, se estableció una lista con distintos motivos para cometer una infidelidad (véase la tabla 1). Para la obtención de estos datos se escogieron únicamente las respuestas de aquellos participantes que habían sido infieles a sus parejas, constituyendo el $27,5 \%$ de la muestra El motivo más prevalente fue «el vínculo emocional era muy poco intenso, no estabas enamorad@» $(38,5 \%)$, seguido de «vuestra relación no iba bien, estabas cansado de la relación» (32,7\%). En cuanto a las diferencias de género (véase la tabla 1 ), solo se establecieron diferencias significativas, a favor de las mujeres, en el motivo «aunque la relación iba bien, no fuiste capaz de controlarte porque habías bebido o tomado alguna droga» $\left(x^{2}=6,422 ; p \leq, 011\right)$. 
Tabla 1

Prevalencia de los motivos para una infidelidad y diferencias de género

\begin{tabular}{|c|c|c|c|c|c|}
\hline \multicolumn{6}{|c|}{ Motivos para una infidelidad } \\
\hline & $\begin{array}{l}\text { Total } \\
(\%)\end{array}$ & $\begin{array}{l}\text { Hombres } \\
(\%)\end{array}$ & $\begin{array}{c}\text { Mujeres } \\
(\%)\end{array}$ & $X^{2}$ & $\mathrm{~V}_{\text {cramer }}$ \\
\hline $\begin{array}{l}\text { El vínculo emocional era muy poco intenso, } \\
\text { no estabas enamorad@ }\end{array}$ & 38,5 & 41,4 & 34,8 & 0,236 & 0,067 \\
\hline $\begin{array}{l}\text { Vuestra relación no iba bien, estabas cansado } \\
\text { de la relación }\end{array}$ & 32,7 & 31,0 & 34,8 & 0,082 & 0,040 \\
\hline $\begin{array}{l}\text { Surgió la posibilidad y no le diste mayor } \\
\text { importancia al hecho }\end{array}$ & 25,5 & 24,1 & 27,3 & 0,065 & 0,036 \\
\hline $\begin{array}{l}\text { Aunque la relación iba bien, no fuiste capaz } \\
\text { de controlarte porque habías bebido o tomado } \\
\text { alguna droga }\end{array}$ & 19,2 & 6,9 & 34,8 & $6,422^{*}$ & 0,332 \\
\hline $\begin{array}{l}\text { Era una venganza porque te había sido infiel } \\
\text { antes }\end{array}$ & 15,4 & 13,8 & 17,4 & 0,128 & 0,049 \\
\hline $\begin{array}{l}\text { Estabas muy excitado y no pudiste controlar } \\
\text { tus impulsos }\end{array}$ & 11,5 & 10,3 & 13,0 & 0,092 & 0,042 \\
\hline $\begin{array}{l}\text { Aunque la relación iba bien, no fuiste capaz } \\
\text { de controlarte porque la otra persona era muy } \\
\text { atractiva }\end{array}$ & 11,5 & 13,8 & 8,7 & 0,327 & 0,079 \\
\hline $\begin{array}{l}\text { Era una forma de castigar a tu pareja por algo } \\
\text { que te había molestado }\end{array}$ & 5,8 & 6,9 & 4,3 & 0,153 & 0,054 \\
\hline $\begin{array}{l}\text { Era un modo de ponerle celoso y que de ese } \\
\text { modo se interesara más por ti }\end{array}$ & 3,8 & 3,4 & 4,3 & 0,028 & 0,023 \\
\hline
\end{tabular}

Nota. ${ }^{*} p<, 05 ;{ }^{* *} p<, 01 ;{ }^{* * *} p<, 001$

\section{Prevalencia de las distintas reacciones ante una infidelidad}

La reacción más común ante una infidelidad sería cortar la relación $(63,6 \%)$, seguido de hablar para saber qué ha fallado y pensar que la relación podría incluso mejorar $(20,7 \%)$ (véase la tabla 2). En cuanto al análisis de las diferencias de género, no se establecieron diferencias significativas en las reacciones ante una infidelidad, observando que ambos sexos tendrían reacciones similares. 
Tabla 2.

Prevalencia de las reacciones ante una infidelidad y diferencias de género

\begin{tabular}{lccccc}
\hline \multicolumn{5}{c}{ Reacciones ante una infidelidad } \\
\hline & $\begin{array}{c}\text { Total } \\
(\%)\end{array}$ & $\begin{array}{c}\text { Hombres } \\
(\%)\end{array}$ & $\begin{array}{c}\text { Mujeres } \\
(\%)\end{array}$ & $X^{2}$ & $\mathrm{p}$ \\
& 63,6 & 63,7 & 63,4 & 0,136 &, 987 \\
$\begin{array}{l}\text { Cortar la relación } \\
\begin{array}{l}\text { Hablarías para saber qué ha fallado y } \\
\text { creerías que la relación podría incluso llegar } \\
\text { a mejorar }\end{array}\end{array}$ & 20,7 & 19,8 & 21,5 & & \\
$\begin{array}{l}\text { Mantener la relación, pero ya no sería lo } \\
\text { mismo }\end{array}$ & 12,5 & 13,2 & 11,8 & \\
$\begin{array}{l}\text { Mantener la relación y perdonar la infidelidad } \\
\text { del todo }\end{array}$ & 3,3 & 3,3 & 3,2 & \\
\hline
\end{tabular}

\section{Relaciones entre variables}

Por último, se llevaron a cabo análisis de correlación para ver si se establecían relaciones entre las distintas variables de estudio. Solo se encontró una relación positiva y significativa entre ser infiel y ser víctima de una infidelidad $(r=0,259 ; p \leq, 000)$.

\section{Discusión}

En la presente investigación se ha observado que la prevalencia de la infidelidad en comparación con otros estudios (Allen y Baucom, 2006; Mcanulty y Brineman, 2007) es relativamente baja. Dichos estudios señalaban alrededor del $70 \%$ de tasa de infidelidad, mientras que nuestros resultados apuntan al $30 \%$. Estas diferencias se podrían atribuir a la subjetividad de la definición de infidelidad que se encuentra en la base de las respuestas de los participantes.

Por otro lado, no se han encontrado diferencias significativas de género a la hora de cometer una infidelidad, al igual que los resultados obtenidos por Randall y Byers (2003). En cambio, al analizar las diferencias en la prevalencia de la infidelidad en función de la orientación sexual, se observa que las personas con una orientación sexual homosexual son significativamente más infieles que heterosexuales y bisexuales. Otras investigaciones que han estudiado estas diferencias encuentran que son las personas con orientación bisexual las que cometen más infidelidad (Giménez, Ballester, Gil y Edo, 2010), por lo que parece que los resultados referidos a este aspecto no son concluyentes.

Principalmente, los motivos por los que se comete una infidelidad es la fragilidad del vínculo afectivo de la pareja junto con las carencias afectivas de la misma, en consonancia con resultados encontrados en estudios previos (Mark y cols., 2011; Weiser y cols., 2014). En lo referente a las diferencias de género, en los motivos para ser infiel, la literatura no es unánime; en el presente estudio se encuentran escasas diferencias de género en los motivos que llevan 
a cometer una infidelidad, únicamente se observan diferencias significativas respecto a cometer una infidelidad bajo los efectos de las drogas o el alcohol, a favor de las mujeres.

Respecto a las reacciones ante una infidelidad, se ha observado que la reacción más frecuente sería cortar la relación después de ser víctima de una infidelidad, sin encontrar diferencias significativas en función del género, en contraposición con los resultados de investigaciones similares que concluyen que las mujeres reaccionarían ante una infidelidad cortando la relación, mientras que los hombres reaccionarían con violencia (Brand y cols., 2007; Weiser y cols., 2014).

En conclusión, mujeres y hombres no difieren significativamente en los motivos y reacciones ante una infidelidad. En futuras investigaciones, sería necesario analizar con profundidad la influencia de posibles aspectos de la relación de pareja sobre los motivos que llevan a cometer una infidelidad, así como las consecuencias que provoca en la pareja.

\section{Referencias bibliográficas}

Allen, E. S. y Baucom, D. H. (2006). Dating, marital and hypothetical extradyadic involvements: how do they compare? Journal of sex research, 43, 307-317.

Blow, A. J. y Hartnett, K. (2005). Infidelity in committed relationships I: a methodological review. Journal of Marital and Family Therapy, 31, 183-216.

Brand, R. J., Markey, C. M., Mills, A. y Hodges, S. D. (2007). Sex differences in self-reported infidelity and its correlates. Sex Roles, 57, 101-109.

Brogdon, B. L., Fitzwater, A. L. y Johnson, L. C. (2006). Differences in Men's and Women's Perception of Infidelity in Varying Situations. All volumes, 18. http://digitalcommons.unf. edu/ojii_volumes/18

Giménez, C., Ballester, R., Gil, M. D. y Edo, M. T. (2010). Prevalencia de la infidelidad en los jóvenes: influencia del género y la orientación sexual. En L. Abad y J. A. Flores: Emociones y sentimientos: la construcción social del amor (pp. 279-290). Cuenca: Universidad de Castilla-La Mancha.

Haden, S. C. y Hojjat, M. (2006). Aggressive responses to betrayal: Type of relationship, victim's sex, and nature of aggression. Journal of Social and Personal Relationships, 23, 101-116.

Mark, K. P., Janssen, E. y Milhausen, R. R. (2011). Infidelity in heterosexual couples: Demographic, interpersonal, and personality-related predictors of extradyadic sex. Archives of Sexual Behavior, 40, 971-982.

McAnulty, R. D. y Brineman, J. M. (2007). Infidelity in dating relationships. Annual review of sex research, 18, 94-114.

Nagurney, A. y Thornton. (2011). What is infidelity? Perceptions based on biological sex and personality. Psychology Research and Behavior Management, 4, 51-58.

Norona, J. C. (2013). Adolescents' Definitions of Cheating in Romantic Relationships. Tennessee, Fl: University of Tennessee.

Randall, H. E. y Byers, E. S. (2003). What is sex? Students' definitions of having sex, sexual partner, and unfaithful sexual behavior. Canadian Journal of Human Sexuality, 12, 87-96.

Shackelford, T. K. y Goetz, A. T. (2008). Personality, Marital Satisfaction, and Probability of Marital Infidelity. Individual Differences Research, 6, 13-25.

Tidwell, N. D. y Eastwick, P. W. (2013). Sex Differences in Succumbing to Sexual Temptations a Function of Impulse or Control? Personality and Social Psychology Bulletin, 39, 16201633. 
Trivers, R. (1972). Parental investment and sexual selection. En B. Campbell (ed.) Sexual selection and the descent of man: 1871-1971 (pp. 136-179). Chicago: Aldine-Atherton.

Vanegas, J. H. (2011). La dinámica vincular celos-infidelidad. Pensamiento Psicológico, 9, 97102.

Weiser, D. A., Lalasz, C. B., Weigel, D. J. y Evans, W. P. (2014). A prototype analysis of infidelity. Personal Relationships, 21, 655-675. 